\title{
Oral Transmucosal Delivery for Improved Drug Bioavailability and Patient Compliance
}

\section{Kiran K Vangara ${ }^{1 *}$ and Sushma Samala ${ }^{2}$}

${ }^{1}$ Research and Development, Insys Therapeutics Inc., Chandler, AZ, USA

${ }^{2}$ Texas A\&M University-Kingsville, Kingsville, TX, USA

Since last 15 years the number of 505(b)(2) filings with new dosage forms for existing drug molecules at United states Food and Drug Administration (USFDA) has been increased tremendously. This is because of the tremendous increase in the developmental cost of new chemical entities (NCE) and increased concerns about patient preferences and market demands for the novel unconventional dosage forms. These new dosage forms are being developed with improved safety and efficacy together with patient compliance using novel drug delivery technologies [1]. Oral transmucosal drug delivery is rapidly growing as a viable alternative for conventional oral dosage forms such as tablets, capsules, syrups and suspensions due to their obvious advantage like delivering drugs directly into the systemic circulation, avoiding first pass drug metabolism, improved bioavailability, low dose related side effects, non-invasiveness, and lack of discomfort [2]. This editorial addressed briefly on current trend of marketed oral transmucosal drug delivery technologies such as sublingual sprays, sublingual tablets, rapid disintegrating tablets, rapid dissolving films/ wafers, medicated chewing gums, and medicated lollipops. These technologies offer better patient compliance over conventional oral dosage forms especially in patients with difficulties in swallowing as they readily dissolve and/or readily deliver drug in mouth cavity. These technologies are best choice for the systemic delivery of drug molecules sensitive to gastrointestinal $\mathrm{pH}$.

\section{Sublingual Delivery}

Sublingual mucosa is the most permeable region in the oral cavity. It is only $100-200 \mu \mathrm{m}$ thick, non-keratinized and highly vasculated. Two dosage forms, sublingual spray and sublingual tablet are available for sublingual delivery. Sublingual spray technology is gaining high attention in pharmaceutical market due its simplicity and ease of administration by patients. Especially where rapid onset of action required i.e. in less than $15 \mathrm{~min}$. In case of sublingual spray, the drug is readily available in dissolved form and upon spray as fine mist, rapidly absorbs into systemic circulation [3]. Rapid pharmacological response is highly desirable in certain cases such as breakthrough pain. Examples: Subsys, a fentanyl unit dose sublingual spray for the treatment of breakthrough cancer pain in opioid-tolerant adult patients. Sativex, a cannabinoid medicine for the symptomatic relief of neuropathic pain in multiple sclerosis in adults. Nitromist ${ }^{\oplus}$, a nitroglycerin spray for the treatment of angina pectoris. Sublingual tablets are compressed with super disintegrants and lower hardness for rapid disintegration under the tongue. Few examples of marketed sublingual tablets include Abstral', Subutex", Aviten', Suboxone', Temgesic', and Nitrostat ${ }^{\circ}[4]$.

\section{Rapid Dissolving Medications}

Rapid mouth dissolving technologies are another possible alternative for improved bioavailability of pharmaceuticals while allowing convenient dosing in paediatric, geriatric and psychiatric patients with dysphagia. They disintegrate or disperse in seconds (less the $30 \mathrm{sec}$ ) without chewing or the need of water and are thus assumed to improve patient compliance. First oral disintegrating or dispersible tablet (ODT) was approved by USFDA in 1996. Since then many
ODTs has been approved for different indications. The ODT market is expected to exceed $\$ 13$ billion by 2015 [5]. FDA defines ODT as "a solid dosage form which contains a medicinal substance or active ingredient which disintegrates rapidly within a matter of seconds when placed upon a tongue". Various techniques such as direct compression, wet granulation, spray-drying, freeze-drying and sublimation were used to prepare ODTs. Novel advanced technologies like Orasolv", Durasolv', Wowtab', Flashtab', Zydis', Flashdose', Oraquick, Lyoc', Advatab, Frosta, Quick-Disc and Nanomelt are introduced by pharmaceutical companies for the preparation of ODTs. Widely used super disintegrants in ODTs include crospovidone, croscaremellose sodium, sodium starch glycolate, and sodium alginate [6].

Rapid dissolving oral film is an emerging new technology slowly talking over ODTs market. Oral therapeutics films (OTFs) are made of easily dispersible hydrophilic polymers and are designed to dissolve upon contact with a wet surface such as the tongue within a few seconds, meaning the consumer can take the product without need for additional liquid. Natural polymers used for developing oral films include pullulan, starch, gelatin, pectin, sodium alginate, and maltodextrins, whereas synthetic polymers used include hydroxypropyl methylcellulose, sodium carboxymethylcellulose, polyethylene oxide, hydroxypropyl cellulose, polyvinylpyrrolidone, and polyvinyl alcohol. The major disadvantages of OTFs are a) drug loading greater than $30 \mathrm{mg}$ is not possible. b) drug should have good solubility in water. Examples: Zuplenz, ondansetron oral film, Listerine ${ }^{\circ}$, Suppress ${ }^{\circ}$, Theraflu', Sudafed $\mathrm{PE}^{\circ}$, Triaminic, and Benadryl $[7,8] \cdot$ Oral films meant for sublingual administration are also available in the market for improved bioavailability. Example: Suboxone, a buprenorphine/naloxone sublingual film. Packaging of oral films is very crucial and films are wrapped individually to prevent moisture pickup. Therapeutic wafer (TF) is the term occasionally used with OTF or ODT developed by lyophilization technology. They are very porous in nature making them to dissolve more quickly in mouth. Examples: Maxalt RPD (rizatriptan wafers), Ondaz-Zydis (Ondansetron wafer), and Klonopin wafers (clonazepam ODT).

\section{Medicated Confectionaries}

Confectionaries such as chewing gums (CG), lozenges and

*Corresponding author: Kiran K Vangara, Research and Development Insys Therapeutics.Inc., Chandler, AZ, USA, Tel: +1602-910-2617; E-mail: vangara.kiran@gmail.com

Received November 26, 2014; Accepted November 26, 2014; Published November 27, 2014

Citation: Vangara KK, Samala S (2015) Oral Transmucosal Delivery for Improved Drug Bioavailability and Patient Compliance. J Bioequiv Availab 7: e58. doi:10.4172/jbb.10000e58

Copyright: (c) 2015 Vangara KK, et al. This is an open-access article distributed under the terms of the Creative Commons Attribution License, which permits unrestricted use, distribution, and reproduction in any medium, provided the original author and source are credited. 
Citation: Vangara KK, Samala S (2015) Oral Transmucosal Delivery for Improved Drug Bioavailability and Patient Compliance. J Bioequiv Availab 7: e58. doi:10.4172/jbb.10000e58

Page 2 of 2

lollipops are popular in food industry and nutraceuticals. Since last few years adding active pharmaceuticals to these confectionaries has been gaining attention of pharmaceutical industry as convenient alternative modified release drug delivery systems especially for pediatric population. These confectionery-based dosage forms have strong potential to overcome compliance problems and are popular amongst the young generation $[9,10]$. Medicated chewing gums (MCG) additionally contain pharmacologically active ingredients along with masticatory gum and excipients such as plasticizer, sweetener, flavor, and/or antioxidants. First MCG "Aspergum" with active ingredient acetyl salicylic acid" was launched in 1928. Other commercially available MCGs include Nicorette and Quitt (contains nicotine for smoking cessation), Travvel (contains dimenhydrinate for motion sickness) [11]. Pharmaceutical companies such as Skypharma, Cargill, Fertin pharma have expertise in developing MCGs.

Medicated lollipop is another emerging technology. MLs are similar in composition to the medicated lozenges with additional stick to hold. Similar to the MCGs they release drug slowly as patient sucks or rotates in the mouth and acts locally or systemically after absorption by buccal/ sublingual/gastrointestinal mucosa. Example: Actiq (contains fentanyl citrate) for the treatment of breakthrough cancer pain in patients of $\geq 16$ years of age. All these formulations discussed above are meant for delivery of drug in oral cavity and most of the active pharmaceuticals possess very bitter or unpleasant taste. So masking unpleasant taste in this formulation is very challenging. One or few combinations of sweetening agent, flavoring agent, taste maker, flavor enhancer can be used to give pleasant taste for better product acceptance.

\section{References}

1. Popa G, Gafitanu E (2003) Oral disintegrating tablets. A new, modern, solid dosage form. Rev Med Chir Soc Med Nat lasi 107: 337-342.

2. Campisi G, Paderni C, Saccone R, Di Fede O, Wolff A, et al. (2010) Human buccal mucosa as an innovative site of drug delivery. Curr pharm des 16: 641652

3. Reisfield GM, Wilson GR (2007) Rational use of sublingual opioids in palliative medicine. J Palliat Med 10: 465-75.

4. 4. Narang N, Sharma J (2011) Sublingual Mucosa as A Route for Systemic Drug Delivery. Int J Pharm Pharm Sci 3: 18-22.

5. Fulzele S, Moe D, Hamed E (2012) Lyoc (Lyophilized Wafer): An Orally Disintegrating Tablet Technology. Drug Development \& Delivery.

6. Badgujar BP, Mundada AS (2011) The technologies used for developing orally disintegrating tablets: a review. Acta pharmaceutica 61: 117-139.

7. Bala R, Pawar P, Khanna S, Arora S (2013) Orally dissolving strips: A new approach to oral drug delivery system. Int J Pharm Investig 3: 67-76.

8. Dixit RP, Puthli SP (2009) Oral strip technology: overview and future potential Journal of controlled release : official journal of the Controlled Release Society 139: 94-107.

9. Chaudhary SA, Shahiwala AF (2010) Medicated chewing gum--a potential drug delivery system. Expert opin Drug Deliv 7: 871-85.

10. Tangso KJ, Ho QP, Boyd BJ (2014) Confectionery-based Dose Forms. Curr Drug Deliv

11. Shah KR, Mehta TA (2014) Medicated Chewing Gum- A Mobile Oral Drug Delivery System. International Journal of Pharm Tech Research 6: 35-48. 